\title{
The effects of digital media on neurodevelopment of children between 18 months and 3 years
}

\author{
Keziban Toksoy 1, Kıvılcım Gucuyener 2, Sebnem Soysal Acar 2 \\ 1.Gazi University, Medical Faculty, Department of Pediatric Neurology, Ankara ,Turkey \\ 2.Gazi University, Medical Faculty, Department of Pediatrics, Ankara ,Turkey
}

\section{Aim-Background}

The period in which neuroplasticity is the fastest and most sensitive to external factors is early childhood. Today's children have more access to electronic media than those of any previous generation. The AAP believing that there were significantly more potential negative effects of media than positive ones for children under 2 years recommended that "pediatricians should urge parents to avoid television viewing for children under the age of two years. High levels of screen time in early childhood also appear to negatively impact academic and social outcomes in the long-term. The objective of this study was to examine the exposure of infants aged between 18- 36 months to television, use of mobile media devices and determine whether duration and content of this exposure is associated with any form of neurodevelopmental regression

\section{Methods - Statistical Analysis}

I In our descriptive and cross-sectional study, 250 children between the ages of 18 months and 3 years who applied to Gazi University Medical Faculty, Pediatric Neurology Clinic between ( June 2017-december 9)were randomly included.Sociodemographic information, digital media usage of parents and their children's were surveyed by a questionnaire : mainly asking; whether parents have media tools such as television, computer / tablet and mobile phone, and how long they spend on such devices, how long the child has been exposed to digital media, what digital media the child used, and how long they used these products. The development of the child in the fine - coarse motor skills, language, and cognitive (social skills - self-care skills) areas was evaluated by the Denver-Il neurodevelopmental screening test.

For the analysis of the data obtained in the study, the SPSS version 20.00 was used. Descriptive analyzes were used to determine frequency, mean, and standard deviation and distribution of data was evaluated by Kolmogorov Smirnov test. Differences between groups was analyzed by ANOVA test, difference between the groups with equal variance was analyzed by Tukey test and the Dunnet test was used when the variances between groups were not equal. Independent $t$ test was used for parametric analysis of gender related data. Chi-Square analysis was used for the analysis of categorical data. The significance level of all statistical analyzes was accepted as $p<0.05$.

\section{Results}

According to the data 158 (64\%) children were reported that they watch TV less than 2 hours, 67 (27\%), 2 - 4 hours, $20(8 \%)$ 4 - 6 hours, 3 (\%1) 6 hours; 133 children (47\%) spend less than 2 hours on a computer / tablet, 91 (36\%) 2 - 4 hours, 40 (16\%) 4 to 6 hours, and 1 child spends more than 6 hours.
As the education level of the mothers increased, the usage time of television, computer / tablet and mobile phone decreased.There was no statistically significant relationship between the fathers' education level and the duration of the child's digital media usage. The longer the parent watched the television, the longer the child watched the television. There was no statistically significant relationship between parents' use of social media and children's computer / tablet usage durations. The frequency of language, cognitive, motor neurodevelopmental retardation was higher in children who had more than 4 hours of television watching, but was lower in children using less than 4 hours of computer-tablet. The frequency of language, fine motor and coarse motor development was worse in children who have used a mobile phone for more than 4 hours a day $(p<0.05)$.

\section{Discussion}

The mount of children's use of digital media tools was found to be lower as maternal education level increased. This suggests that mothers with high level of education are aware of the children's digital media usage. Recurrent experiences show progress in both the fine motor and cognitive development of the children who have the opportunity to recognize and learn the environment and its objects. Motor development of children who spend more time watching television and playing with cell phones instead of active games is adversely affected. In our study; the frequency of language, cognitive, motor neurodevelopmental retardation was higher in children who had more than 4 hours of television watching, but was lower in children using less than 4 hours of computer-tablet. The frequency of language, fine motor and coarse motor development was worse in children who have used a mobile phone for more than 4 hours a day. In addition to the cognitive skills of the child who has less communication with their environment, the linguistic skills are also left behind.

\section{Conclusion}

By the advances of technology, digital media tools have become a part of everyday life. In our study, digital media usage of children aged 18 months - 3 years were found to be quite high. Increasing television screentime negatively effects neuroneurodevelopmental status. Public health interventions will be necessary to optimize neurodevelopmental outcomes

\section{References}

Helena Duch,Elisa M Fisher,Ipek Ensari,Alison Harrington ,Screentime use in children under 3 years old:a systematic review of correlates, Duch et al. International Journal of Behavioral Nutrition and Physical Activity 2013, Radesky J.S., Christakis D.A. Increased Screen Time Implications for Early Chilhood Development and Behavior: Pediatr Clin North Am. 2016;63:827-39 